\title{
Atuação fonoaudiológica em pacientes com câncer de esôfago submetidos a tratamento radioterápico: Série de casos
}

\author{
Speech therapy performance in patients \\ with esophagus cancer receiving radiotherapy \\ treatment: A case series
}

\section{Actuación fonoaudiológica en pacientes con cáncer de esófago en radioterapia: Serie de casos}

\author{
Bárbara Luísa Simonetti* (D) \\ Vera Beatris Martins* (D) \\ Monalise Costa Batista Berbert*
}

\section{Resumo}

Introdução: A atuação fonoaudiológica na disfagia decorrente do câncer de esôfago ainda é recente. Não foi encontrado até o momento, estudos que descrevam a atuação fonoaudiológica em pacientes com câncer de esôfago submetidos a radioterapia. Sendo assim, este trabalho teve como objetivo descrever a atuação fonoaudiológica em pacientes com câncer de esôfago, submetidos a tratamento radioterápico com queixa de disfagia. Métodos: Trata-se de uma série de cinco casos, que receberam acompanhamento fonoaudiológico ambulatorial. Os pacientes foram avaliados por meio de avaliação clínica da deglutição, classificação do grau de alteração de deglutição com aplicação de escala visual analógica e protocolos de qualidade de vida utilizados na área da oncologia. Foram submetidos ainda a uma abordagem terapêutica para deglutição. Resultados: Os pacientes obtiveram melhora da deglutição, apresentando, ao término do tratamento, diminuição dos sintomas de disfagia, possibilidade de ingesta de alimentos via oral e melhora da qualidade de vida. Conclusão: A fonoterapia, compreendendo a avaliação clínica da deglutição, seguida

* Universidade Federal de Ciências da Saúde de Porto Alegre, RS, Brasil.

Contribuição dos autores:

BLS: Concepção do estudo; Metodologia; Coleta de dados; Esboço do artigo.

VBM e MCBB: Esboço do artigo; Revisão crítica; Orientação.

E-mail para correspondência: Bárbara Luísa Simonetti - barbaralsimonetti@hotmail.com Recebido: $19 / 06 / 2020$

Aprovado: 02/12/2020 
de treino da deglutição e exercício miofuncional para abertura do esfíncter esofágico superior (Shaker), auxiliou na reabilitação da disfagia esofágica.

Palavras-chave: Fonoaudiologia; Neoplasias Esofágicas; Radioterapia; Transtornos de deglutição; Qualidade de vida.

\section{Abstract}

Introduction: Speech therapy performance in dysphagia resulting from esophageal cancer is still a recent subject. To date, there are no studies describing the speech therapy performance of patients with esophageal cancer undergoing radiotherapy. For this reason, the aim of this study was to describe speech therapy performance in patients with esophageal cancer receiving radiotherapy treatment and with complaints of dysphagia. Methods: This is a series of five cases, with patients that received outpatient speech therapy. The patients were assessed using clinical swallowing evaluation, classification of the degree of swallowing alteration with application of visual analog scale and quality of life protocols used in oncology. They also underwent a therapeutic approach to swallowing. Results: The patients' swallowing was improved and, at the end of the treatment, there were reduced symptoms of dysphagia, possibility of oral intake of food and improved quality of life. Conclusions: Speech therapy, comprising the clinical assessment of the swallowing function, followed by swallowing training and myofunctional exercise to open the upper esophageal sphincter, supported the rehabilitation of esophageal dysphagia.

Keywords: Speech, Language and Hearing Sciences; Esophageal Neoplasms; Radiotherapy; Deglutition Disorders; Quality of Life.

\section{Resumen}

Introducción: La actuación fonoaudiológica en disfagia decurrente de cáncer del esófago es todavía reciente. Hasta ahora, no se han encontrado estudios que describan la actuación fonoaudiológica en pacientes con cáncer de esófago sometidos a radioterapia. De esta forma, este trabajo tuvo como objetivo describir la actuación del fonoaudiologo en pacientes con cáncer de esófago sometidos a tratamiento de radioterapia con queja de disfagia.Métodos: Esta es una serie de cinco casos, que recibieron terapia fonoaudiologica en ambulatorio. Los pacientes fueron evaluados mediante evaluación clínica de deglución, clasificación del nivel de alteración de la deglución con aplicación de escala visual analógica y protocolos de calidad de vida utilizados en el área de oncología. También fueron sometidos a un enfoque terapéutico para la deglución. Resultados: Los pacientes mejoraron su deglución, presentando, al final del tratamiento, una reducción en los síntomas de disfagia, la posibilidad de ingesta de alimentos por vía oral y una mejora en la calidad de vida. Conclusión: La terapia fonoaudiologica, que comprende la evaluación clínica de deglución, seguida del entrenamiento de deglución y ejercicio miofuncional para abrir el esfínter esofágico superior, ayudó en la rehabilitación de la disfagia esofágica.

Palabras clave: Fonoaudiología; Neoplasias Esofágicas; Radioterapia; Trastornos de Deglución; Calidad de Vida.

\section{Introdução}

O câncer de esôfago é uma neoplasia maligna de início insidioso, altamente agressivo e com prognóstico desfavorável. No Brasil, é considerada a $6^{\mathrm{a}}$ neoplasia mais frequente entre os homens e a $15^{\mathrm{a}}$ entre as mulheres, exceto câncer de pele não melanoma. Estima-se que para cada ano do biênio (2018-2019), haverá 8.240 (3,8\%) novos casos de câncer de esôfago em homens e $2.550(1,3 \%)$ em mulheres. ${ }^{1}$
Fatores como extensão da doença, condições clínicas do paciente e tipo específico de tumor vão determinar a modalidade terapêutica a ser empregada. Nos casos de doença ressecável, são indicados quimioterapia, radioterapia, ressecção cirúrgica e/ou associação destas modalidades. Frequentemente, tem sido utilizadas a quimioterapia e radioterapia neoadjuvante, seguidas de cirurgia. ${ }^{2}$

Indivíduos com câncer de esôfago podem apresentar em maior ou menor grau, alterações de deglutição, seja pela sintomatologia causada pelo 
próprio tumor, como por exemplo, sensação de alimento parado na transição faringoesofágica pela obstrução na passagem do alimento, e/ou pelo tipo de tratamento submetido, como por exemplo radioterapia, quimioterapia e/ou cirurgia. Uma revisão sistemática recente identificou que a reabilitação da disfagia orofaríngea após esofagectomia apresenta eficácia por meio de exercícios de respiração freno-labial, amplitude do movimento cervical (cabeça inclinada para frente, para trás e para os lados), alongamento de ombro, abertura de mandíbula, exercícios isométricos e isotônicos de língua (protrusão, retração, elevação e abaixamento), treinamento da musculatura supra-hióidea (exercício de Shaker na forma de apresentação isométrica e isotônica), além da utilização de manobras de proteção de vias aéreas, como é o caso da manobra de queixo para baixo durante a deglutição. ${ }^{3}$ Entretanto, não foram encontradas, até o momento, descrições da abordagem terapêutica em relação ao atendimento fonoaudiológico de pacientes com disfagia esofágica submetidos exclusivamente a tratamento combinado (quimioterapia e radioterapia).

Diante deste contexto de escassez de publicações sobre o tema, os relatos de casos tornam-se necessários para iniciar o processo de ampliação, aprofundamento e produção de evidências científicas sobre as abordagens terapêuticas utilizadas na reabilitação fonoaudiológica de pacientes com câncer de esôfago. Tal fato justifica a importância deste estudo, cujo objetivo é descrever a atuação fonoaudiológica em pacientes com câncer de esôfago, com queixa de disfagia, durante o tratamento radioterápico.

\section{Apresentação dos casos clínicos}

Trata-se de um estudo do tipo relatos de casos, descritivo, aprovado pelo Comitê de Ética em Pesquisa da instituição, sob o parecer de número 3.109.023. Todos os participantes assinaram o Termo de Consentimento Livre e Esclarecido.

A amostra foi composta por indivíduos adultos, diagnosticados com câncer de esôfago, que haviam sido submetidos a tratamento de radioterapia combinada ou não com quimioterapia, no período de abril a julho de 2019, que apresentassem queixa de disfagia, encaminhados para o serviço de Fonoaudiologia. Foram excluídos pacientes submetidos a procedimentos cirúrgicos na região faringoesofágica, que possuíssem histórico prévio de outras neoplasias, e que apresentassem metástases, para enfoque mais específico das questões esofágicas. De acordo com estes critérios, foram selecionados cinco casos para descrição.

- Caso 1 - Sexo feminino; 79 anos; viúva; aposentada; três anos de escolaridade; hipertensa; não etilista e/ou tabagista; histórico familiar de câncer de laringe (mãe). Procurou atendimento médico com queixa de dor abdominal após alimentação e perda de peso; diagnosticada com adenocarcinoma invasor de esôfago distal; com indicação de radioterapia e quimioterapia. Alimentava-se através de sonda nasoentérica (SNE) exclusiva.

- Caso 2 - Sexo feminino; 45 anos; casada; dona de casa; 11 anos de escolaridade; sem comorbidades; não etilista e/ou tabagista; histórico familiar de câncer de útero (mãe e irmã). Procurou atendimento médico com queixa de disfagia, odinofagia e perda de peso; diagnosticada com carcinoma espinocelular de esôfago distal; indicação de radioterapia e quimioterapia e, cirurgia após tratamento clínico combinado. Alimentava-se através de SNE exclusiva.

- Caso 3 - Sexo feminino; 84 anos; casada; aposentada; quatro anos de escolaridade; hipertensa; diabética; não etilista e/ou tabagista; sem histórico familiar de câncer. Procurou atendimento médico com queixa de disfagia para sólidos, perda de peso e histórico de úlcera gástrica recente; diagnosticada com adenocarcinoma de esôfago distal. Com indicação de radioterapia exclusiva. Alimentava-se por via oral, na consistência pastosa.

- Caso 4 - Sexo masculino; 60 anos; divorciado; aposentado; um ano de escolaridade; cardiopata; pneumopata; ex-etilista e ex-tabagista; histórico familiar de câncer de estômago (avô). Procurou atendimento médico com queixa de disfagia para sólidos e perda de peso; diagnosticado com carcinoma epidermóide de esôfago medial; indicação de quimioterapia e radioterapia. Alimentava-se por via oral, na consistência semi-sólida.

- Caso 5 - Sexo masculino; 68 anos; casado; aposentado; cinco anos de escolaridade; apresentando comorbidades como cirrose alcoólica; aneurisma de ilíacas à esquerda; insuficiência cardíaca à direita; fibrilação atrial; soropositivo. Ex-etilista e ex-tabagista; histórico familiar de câncer de estômago (irmão) e câncer de mama (filha). Procurou atendimento médico devido disfagia para sólidos e perda de peso. Diagnosticado 
com carcinoma epidermóide pouco diferenciado invasor de esôfago medial. Indicação de quimioterapia e radioterapia. Alimentava-se por via oral, na consistência semi-sólida.

Os pacientes receberam acompanhamento fonoaudiológico semanal, com sessões de $40 \mathrm{mi}-$ nutos, por uma mesma fonoaudióloga, durante todo o tratamento de radioterapia. O serviço apresenta rotina de protocolos de avaliação descritos a seguir.

Todos os pacientes foram submetidos a avaliação indireta da deglutição, na qual avaliou-se sensibilidade, mobilidade, tônus e função dos órgãos fonoarticulatórios, e a avaliação direta da deglutição, com diferentes consistências alimentares, a fim de verificar o inventário oral de cada paciente, de acordo com um protocolo próprio da Instituição. $\mathrm{O}$ inventário oral foi estabelecido por meio da seguinte classificação ${ }^{4}$ : saliva, líquido fino, líquido levemente espessado (consistência néctar), líquido moderadamente espessado (consistência mel), líquido extremamente espessado (consistência pudim) /pastoso, semi sólido e sólido. Determinou-se o nível de ingestão oral por meio da Functional Oral Intake Scale (FOIS). ${ }^{5}$

Para classificar o grau de alteração da deglutição utilizou-se a Escala Visual Analógica (EVA). Esta escala pontua de zero a dez esta alteração, sendo dez o valor máximo, equivalente a uma alteração intensa; cinco um valor moderado, equivalente a uma alteração moderada; e, zero um valor nulo, equivalente a nenhuma alteração. Este instrumento possibilita observar a evolução da alteração de deglutição.
Foi avaliada a qualidade de vida relacionada à saúde, por meio dos questionários utilizados na área de oncologia, da European Organization for Research and Treatment of Cancer (EORTC): o EORTC QLQ-C30 e o EORTC QLQ-OES18. O questionário EORTC QLQ-C30, traduzido e validado para o Português Brasileiro, ${ }^{6}$ contém 30 questões de qualidade de vida em geral, e é utilizado para qualquer tipo de câncer. É dividido em três escalas: estado de saúde global e qualidade de vida, funcional e de sintomas. Este instrumento é geralmente utilizado em associação a outros módulos, específicos para cada tumor, que neste estudo foi o de câncer de esôfago.

O questionário EORTC QLQ-OES18, validado para o Português, ${ }^{7}$ contém 18 questões específicas sobre o câncer de esôfago. O instrumento é dividido em duas escalas: funcional e sintomas. Para a interpretação dos resultados dos dois instrumentos, são utilizadas as normas do manual da EORTC, ${ }^{8}$ identificando que quanto maior a escala funcional melhor é a qualidade de vida, enquanto um escore alto na escala de sintoma, representa um nível baixo na tolerância de sintomas e efeitos colaterais.

Foi possível descrever os resultados apresentados pelos pacientes, através dos instrumentos aplicados a cada sessão de fonoterapia, a constar: avaliação clínica da deglutição para determinar o nível de ingestão oral (Tabela 1), as consistências alimentares favoráveis a cada paciente (Tabela 2) e EVA (Tabela 3). Além disso, foram aplicados no início e ao término do tratamento fonoaudiológico e radioterápico os protocolos de qualidade de vida: QLQ-C30 versão 3.0 (Tabela 4) e QLQ-OES18 versão 2.0 (Tabela 5).

Tabela 1. Nível de ingestão oral a cada sessão de fonoterapia - Escala FOIS

\begin{tabular}{cccccccc}
\hline Caso & $\mathbf{1}^{\mathbf{a}}$ sessão & $\mathbf{2}^{\mathbf{a}}$ sessão & $\mathbf{3}^{\mathbf{a}} \mathbf{~ s e s s a ̃ o ~}$ & $\mathbf{4}^{\mathbf{a}}$ sessão & $\mathbf{5}^{\mathbf{a}}$ sessão & $\mathbf{6}^{\mathbf{a}} \mathbf{~ s e s s a ̃ o ~}$ & $\mathbf{7}^{\mathbf{a}}$ sessão \\
\hline C1 & 1 & 2 & 2 & 3 & 3 & 3 & - \\
C2 & 1 & 1 & 2 & 2 & 2 & 2 & - \\
C3 & 5 & 5 & 3 & 3 & 3 & 3 & - \\
C4 & 6 & 6 & 6 & 7 & 7 & 7 & - \\
C5 & 6 & 6 & 6 & 6 & 7 & 7 & 7
\end{tabular}

Legenda: C - caso. 
Tabela 2. Evolução da consistência alimentar a cada sessão de fonoterapia

\begin{tabular}{|c|c|c|c|c|c|c|c|}
\hline Caso & $\begin{array}{c}1^{a} \\
\text { sessão }\end{array}$ & $\begin{array}{c}2^{a} \\
\text { sessão }\end{array}$ & $\begin{array}{c}3^{a} \\
\text { Sessão }\end{array}$ & $\begin{array}{c}4^{a} \\
\text { sessão }\end{array}$ & $\begin{array}{c}5^{a} \\
\text { sessão }\end{array}$ & $\begin{array}{c}6^{a} \\
\text { Sessão }\end{array}$ & $\begin{array}{c}7^{a} \\
\text { sessão }\end{array}$ \\
\hline $\mathrm{C} 1$ & Saliva & Líquido fino & Néctar & $\begin{array}{l}\text { Pudim/ } \\
\text { Pastosa }\end{array}$ & $\begin{array}{l}\text { Pudim/ } \\
\text { Pastosa }\end{array}$ & Semi-sólido & - \\
\hline $\mathrm{C} 2$ & NPO & Saliva & Líquido fino & Néctar & Néctar & Mel & - \\
\hline C3 & $\begin{array}{l}\text { Pudim/ } \\
\text { Pastosa }\end{array}$ & $\begin{array}{l}\text { Pudim/ } \\
\text { Pastosa }\end{array}$ & $\begin{array}{l}\text { Pudim/ } \\
\text { Pastosa }\end{array}$ & Mel & Mel & Mel & - \\
\hline $\mathrm{C} 4$ & Semi-sólido & Semi-sólido & Semi-sólido & Sólidos & Sólidos & Sólidos & - \\
\hline C5 & Semi-sólido & Semi-sólido & Semi-sólido & Semi-sólido & Sólidos & Sólidos & Sólidos \\
\hline
\end{tabular}

Legenda: C - caso; NPO - nada por via oral.

Tabela 3. Escala visual analógica a cada sessão de fonoterapia

\begin{tabular}{cccccccc}
\hline Caso & $\mathbf{1}^{\mathbf{a}}$ sessão & $\mathbf{2}^{\mathbf{a}}$ sessão & $\mathbf{3}^{\mathbf{a}}$ sessão & $\mathbf{4}^{\mathbf{a}}$ sessão & $\mathbf{5}^{\mathbf{a}}$ sessão & $\mathbf{6}^{\mathbf{a}}$ sessão & $\mathbf{7}^{\mathbf{a}}$ sessão \\
\hline C1 & 10 & 5 & 4 & 2 & 2 & 2 & - \\
C2 & 10 & 7 & 5 & 2 & 2 & 2 & - \\
C3 & 4 & 6 & 6 & 7 & 7 & 7 & - \\
C4 & 2 & 2 & 2 & 0 & 0 & 0 & - \\
C5 & 4 & 2 & 2 & 3 & 2 & 2 & 0 \\
\hline
\end{tabular}

Legenda: C - caso.

Tabela 4. Escores médios das escalas de qualidade de vida pré e pós fonoterapia e radioterapia, segundo o QLQ-C30

\begin{tabular}{ccccccc}
\hline Escalas do QLQ-C30 & $\begin{array}{c}\text { Período do } \\
\text { tratamento }\end{array}$ & $\mathbf{C 1}$ & $\mathbf{C 2}$ & $\mathbf{C 3}$ & $\mathbf{C 4}$ & $\mathbf{C 5}$ \\
\hline Estado de saúde global & Antes & 100 & 100 & 75 & 83,33 & 75 \\
e qualidade de vida & Depois & 41,66 & 100 & 75 & 100 & 66,66 \\
Funcionais & Antes & 13,33 & 4,44 & 11,11 & 24,44 & 13,33 \\
& Depois & 31,11 & 8,88 & 57,77 & 17,77 & 6,66 \\
Sintomas & Antes & 10,25 & 15,38 & 17,94 & 15,38 & 5,12 \\
& Depois & 61,53 & 15,38 & 51,28 & 7,69 & 10,25 \\
\hline
\end{tabular}

Legenda: C - Caso.

Tabela 5. Escores médios das escalas de qualidade de vida pré e pós fonoterapia e radioterapia, segundo o QLQ-EOS18

\begin{tabular}{ccccccc}
\hline Escalas do QLQ-EOS18 & $\begin{array}{c}\text { Período do } \\
\text { tratamento }\end{array}$ & C1 & C2 & C3 & C4 & C5 \\
\hline \multirow{2}{*}{ Funcional } & Antes & 11,11 & 11,11 & 33,33 & 77,77 & 66,66 \\
& Depois & 22,22 & 22,22 & 11,11 & 88,88 & 88,88 \\
Sintomas & Antes & 22,22 & 33,33 & 28,88 & 4,44 & 6,66 \\
& Depois & 28,88 & 17,77 & 40 & 2,22 & 2,22 \\
\hline
\end{tabular}

Legenda: C - caso.

Após as avaliações foram realizadas as sessões de terapia. A fonoterapia foi guiada pelos dados das avaliações que apontavam o plano terapêutico. A sessão de terapia iniciava-se pelo processo de escuta do paciente e conscientização dos objetivos do tratamento e de cada procedimento; em seguida era realizada a avaliação direta com o alimento e revisado o exercício para o aperfeiçoamento da realização do gesto motor. Observou-se, em todos os casos, a utilização do exercício de Shaker, na forma de apresentação isométrica e isotônica, ${ }^{9}$ visto que todos os pacientes referiam "sensação de alimento parado" em região de faringe e esôfago. Os pacientes foram orientados a realizar o exercício durante $o$ atendimento e no âmbito domiciliar, três vezes ao dia. Além disso, foi solicitado o preenchi- 
mento de um diário de exercícios que servia para verificar a adesão ao tratamento. Quatro pacientes realizaram o total de seis sessões de fonoterapia, de acordo com o período da radioterapia, e um deles, sete sessões, pois seu tratamento de radioterapia durou sete semanas.

\section{Discussão}

A atuação fonoaudiológica no câncer de esôfago ainda é recente, porém observa-se aumento da demanda e dos encaminhamentos desses pacientes em função da disfagia faríngea e/ou esofágica. Este estudo apresentou uma série de casos e demonstrou que para pacientes com câncer de esôfago em radioterapia, a avaliação clínica deve abranger instrumentos que verifiquem a função de deglutição, especialmente a nível esofágico, e a qualidade de vida.

Histologicamente, o câncer de esôfago pode ser classificado em dois tipos principais: o carcinoma espinocelular e o adenocarcinoma. O primeiro acomete preferencialmente os terços superior e médio do esôfago e tem como fator de risco o etilismo e o tabagismo. Já o segundo se manifesta no terço inferior, podendo resultar em metaplasia intestinal, devido ao refluxo gástrico crônico e tem como fator de risco a obesidade, o hábito de fumar e o esôfago de Barrett. ${ }^{10}$ Esse achado vai ao encontro dos dados coletados dos pacientes descritos nesse relato, em que verificou-se a ocorrência de alterações esofágicas no terço distal em pacientes que não eram tabagistas e etilistas (Casos 1, 2 e 3). Em contrapartida, os dois pacientes que apresentavam alterações esofágicas no terço médio tinham histórico de tabagismo e etilismo (Casos 4 e 5).

Em relação aos sintomas apresentados, houve predomínio da queixa de disfagia para sólidos e perda de peso. Tal dado corrobora com outro estudo ${ }^{11}$ que descreve como sintomas decorrentes do câncer de esôfago: disfagia, odinofagia, desconforto retroesternal, sensação de corpo estranho no esôfago, dor epigástrica, febre, fadiga, náuseas, vômitos após as refeições, desidratação, alteração na qualidade vocal e perda de peso sem causa aparente. Os sintomas apresentados são decorrentes da obstrução esofágica ocasionada pelo tumor neste órgão.

$\mathrm{Na}$ terapia fonoaudiológica observou-se a utilização do exercício de Shaker, na forma de apresentação isométrica e isotônica ${ }^{9}$ em todos os casos relatados, visto que a queixa principal dos pacientes eram disfagia para sólidos e sensação alimento parado na região faringoesofágica. O exercício Shaker já foi utilizado em outros estudos ${ }^{12}$, inclusive com pacientes disfágicos com disfunção do esfíncter esofágico superior (EES), uma vez que o mesmo se baseia no movimento de elevação e anteriorização da laringe, decorrente da tração dos músculos milo-hióideo, tireo-hióideo, genio-hióideo e do ventre anterior do digástrico, resultando na abertura do EES. Infere-se que o exercício orientado, durante o tratamento radioterápico, contribuiu para sanar a queixa de disfagia esofágica. $\mathrm{O}$ atendimento fonoaudiológico semanal e o acompanhamento da realização dos exercícios através do preenchimento de um diário pode ter contribuído para a maior adesão a terapia e, consequentemente, para a melhora dos sintomas de deglutição.

Foi possível observar, nos casos relatados, uma evolução na ingesta de alimento por via oral do início ao término da fonoterapia/radioterapia, quando analisados os resultados da escala FOIS, exceto uma única paciente que necessitou da introdução de SNE para alimentação durante a radioterapia. Este achado demonstra que a terapia fonoaudiológica, associada ao tratamento radioterápico e/ou quimioterápico, contribui para a melhora da ingesta de alimento por via oral, visto que o exercício de fonoterapia visa maximizar a função de deglutição e, consequentemente, auxiliar na motilidade esofágica, ao mesmo tempo que ocorre a diminuição do tumor resultante do tratamento oncológico. É importante ressaltar que, a manutenção da dieta por via oral foi viabilizada no caso da paciente que necessitou fazer uso de SNE, uma vez que foi inserida dieta mista com involução da consistência alimentar como estratégia de compensação, diante de uma pior performance da paciente frente a complicações do tratamento.

Quanto à consistência alimentar, observou-se que no decorrer do tratamento foram necessárias algumas adaptações, que na sua maioria (quatro casos) foi numa crescente progressão da consistência. Somente uma paciente necessitou regredir a consistência da dieta, de uma pastosa para líquidos moderadamente espessados (consistência mel), devido ao mau estado geral decorrente da quimioterapia neoadjuvante. Cabe ressaltar o fato, de que as duas pacientes que apresentavam dificuldade até para deglutição de saliva inicialmente, conseguiram após duas semanas de fonoterapia, ingerir 
líquido fino por via oral. Há descrito na literatura ${ }^{13}$ que os sintomas decorrentes da radioterapia na região do esôfago levam cerca de duas semanas para aparecer, visto que o tempo de latência para resposta do tecido depende das taxas fisiológicas de diferenciação celular, perda e renovação, que neste órgão é mais lento. Sendo assim, pode-se verificar que as pacientes se beneficiaram com fonoterapia antes mesmo da radioterapia começar a causar alterações estruturais.

Da mesma forma que ocorreu a progressão da consistência alimentar, os pacientes referiam melhora para deglutir, no que diz respeito ao conforto, demonstrado na redução dos valores da EVA, sendo que as mudanças mais expressivas foram identificadas a partir da quarta sessão. Diante do exposto, pode-se perceber que a grande maioria dos pacientes que receberam terapia fonoaudiológica durante o tratamento radioterápico mantiveram ou melhoraram sua performance alimentar durante $o$ tratamento, o que difere da prática clínica, em pacientes que não realizam fonoterapia. Na maioria das vezes, permanecem sem via oral além de evoluírem para dificuldades alimentares mais intensas.

A qualidade de vida é outro ponto crucial nestes casos, visto que a taxa de sobrevida global de pacientes com câncer de esôfago é pequena e que estes estão suscetíveis a complicações decorrentes do tratamento neoadjuvante, com quimioterapia e radioterapia. ${ }^{11}$ Analisando os resultados obtidos pelo questionário EORTC QLQ-C30 pôde-se verificar que a maioria dos pacientes apresentou melhora do estado de saúde global e da qualidade de vida ao término do tratamento radioterápico e fonoterápico. No que se refere à escala funcional, houve melhora em grande parte dos pacientes, especialmente, nas questões relacionadas ao estado emocional, condição física e desempenho de papel, visto que, estes pacientes passaram a interagir mais em suas relações sociais quando retomaram e/ou aperfeiçoaram a ingesta de alimento via oral. Em se tratando da escala de sintomas, verificou-se também um aumento na maioria dos casos ao término do tratamento, quando comparados ao início da radioterapia, sendo a fadiga, dor, perda de apetite, náuseas e vômitos os mais prevalentes. Estes, por sua vez, foram apresentados pelos pacientes que realizam o tratamento combinado de radioterapia e quimioterapia. Dado que corrobora com outro estudo ${ }^{14}$ que descreve que os cinco sintomas mais afetados no QLQ-C30, em pacientes submetidos ao mesmo tratamento combinado, em ordem decrescente, foram fadiga, perda de apetite, dor, náusea/ vômitos e dispneia. Em contrapartida, no mesmo estudo, houve declínio significativo da qualidade de vida relacionada à saúde, sendo a condição física e o desempenho de papel as funções mais prejudicadas após o tratamento.

Os resultados do questionário EORTC QLQ-OES18, que contém questões específicas sobre o câncer de esôfago, mostraram uma melhora funcional relacionada à queixa da disfagia, associada a uma melhor qualidade de vida, em quatro pacientes. Achado este que vai ao encontro do que foi apresentado em um outro estudo ${ }^{15}$ que verificou que a disfagia melhorou após o tratamento neoadjuvante. A única paciente que teve piora da disfagia foi a que apresentou um quadro de mau estado geral após sessões de quimioterapia. No que se refere à escala de sintomas, houve diminuição dos sintomas apresentados: alimentação, refluxo e dificuldade para engolir saliva. Identificou-se, ainda, aumento da queixa de dor e problemas com paladar ao término da radioterapia, o que condiz com dados da literatura a respeito dos efeitos adversos da radioterapia. ${ }^{16}$

\section{Conclusão}

Diante do exposto, pode-se concluir que a atuação fonoaudiológica ocorreu de forma precoce, já na primeira sessão da radioterapia, com intervenções terapêuticas que buscaram amenizar os sintomas apresentados e, consequentemente, melhorar a qualidade de vida dos pacientes. A avaliação do impacto funcional e da qualidade de vida devem ser consideradas como fatores importantes, na escolha das modalidades terapêuticas utilizadas no tratamento oncológico, especialmente quando a sobrevida, baseada no controle do tumor, é similar entre si. É através desta avaliação que se pode compreender o grau de melhoria, estabilidade ou piora decorrente do tratamento.

Foi utilizado o exercício de Shaker, na forma de apresentação isométrica e isotônica, em todos os pacientes durante a fonoterapia. A mensuração dos efeitos da terapia fonoaudiológica se deu através da avaliação clínica da deglutição e da aplicação de protocolos de qualidade de vida, utilizados na área de oncologia, da European Organization for Research and Treatment of Cancer (EORTC QLQ-C30 e EORTC QLQ-OES18). Infere-se que 
a intervenção contribuiu para a manutenção da deglutição funcional e para melhora das alterações de deglutição.

Estudos futuros, buscam a utilização de exames objetivos da deglutição, como a videofluoroscopia, para observação de eventos fisiológicos da fase esofágica da deglutição diante de manobras realizadas, para embasamento de posteriores ensaios clínicos com protocolo terapêutico.

\section{Referências}

1. INCA: Instituto Nacional do Câncer [internet]. Rio de Janeiro: Instituto Nacional do Câncer; c1995-2018. Estimativa 2018: Incidência de Câncer no Brasil. [Acesso em 2019 ago. 05]. Disponível em: <http://www1.inca.gov.br/estimativa/2018/ estimativa-2018.pdf $>$.

2. Ruivo EAB, Mello JRC, Cavenaghi OM, Ferreira LL. Perfil sociodemográfico e clínico de pacientes com neoplasia de esôfago e estômago em um hospital escola de São José do Rio Preto, SP, Brazil. Rev Fac Ciênc Méd Sorocaba. 2017; 19(4): $189-95$

3. Kaneoka A, Yang S, Inokuchi H, Ueha R, Yamashita H, Nito T, et al. Presentation of oropharyngeal dysphagia and rehabilitative intervention following esophagectomy: a systematic review. Diseases of the Esophagus. 2018; 31(8): 1-11.

4. Steele CM, Alsanei WA, Ayanikalath S, Barbon CEA, Chen $\mathrm{J}$, Cichero JAY, et al. The Influence of Food Texture and Liquid Consistency Modification on Swallowing Physiology and Function: A Systematic Review. Dysphagia. 2015; 30(1): 2-26.

5. Crary MA, Carnaby-Mann GD, Groher ME. Initial psychometric assessment of a Functional Oral Intake Scale for Dysphagia in stroke patients. Arch Phys Med Rehabil. 2005; 86(8): 1516-20

6. Franceschini J, Jardim JR, Fernandes ALG, Jamnik S, Santoro IL. Reproducibility of the Brazilian Portuguese version of the European Organization for Research and Treatment of Cancer Core Quality of Life Questionnaire used in conjunction with its lung cancer-specific module. J Bras Pneumol. 2010; 36(5): 595-602.

7. Relvas-Silva M, Silva RA, Dinis-Ribeiro M. Portuguese Version of the EORTC QLQ-OES18 and QLQ-OG25 for Health-Related Quality of Life Assessment. Acta Med Port. 2017; 30(1): 47-52.

8. Fayers PM, Aaronson NK, Bjordal K, Groenvold M, Curran D, Bottomley A. EORTC QLQ-C30 Scoring Manual. European Organisation for Research and Treatment of Cancer. 2001; 3(86): $1-78$

9. Shaker R, Kern M, Bardan E, Taylor A, Stewart ET, Hoffmann RG, et al. Augmentation of deglutitive upper esophageal sphincter opening in the elderly by exercise. Am J Physiol. 1997; 272(6): G1518-22

10. Henry MACA, Lerco MM, Ribeiro PW, Rodrigues MAM. Epidemiological features of esophageal cancer. Squamous cell carcinoma versus adenocarcinoma. Acta Cirurgica Brasileira. 2014; 29(6): 389-93
11. Oliveira-Borges EC, Silva AF, Graças AM, Melo FFS, Barcelos AA, Myiata S. O câncer de esôfago: uma revisão. Revista da Universidade Vale do Rio Verde. 2015; 13(1): 773-90.

12. Ferreira TS, Mangilli LD, Sassi FC, Fortunato-Tavares T, Limongi SCO, Andrade CRFD. Speech and myofunctional exercise physiology: a critical review of the literature. $J$ Soc Bras Fonoaudiol. 2011; 23(3): 288-96.

13. Halperin EC, Wazer DE, Perez CA, Brady LW. Perez and Brady's Principles and practice of radiation oncology. 6rd ed. Philadelphia: Lippincott Williams \& Wilkins; 2013.

14. Meerten EV, Gaast AVD, Looman CWN, Tilanus HWG, Muller K, Essink-Bot ML. Quality of life during neoadjuvant treatment and after surgery for resectable esophageal carcinoma. Int. J. Radiation Oncology Biol. Phys. 2008; 71(1): 160-6.

15. Scarpa M, Saadeh LM, Fasolo A, Alfieri R, Cagol M, Cavallin F, et al. Health-related quality of life in patients with oesophageal cancer: Analysis at different steps of the treatment pathway. J Gastrointest Surg. 2013; 17(3): 421-33.

16. Silva AIV, Galante C, Manzi FR. Efeito da radiação ionizante sobre o paladar em pacientes submetidos à radioterapia para a região da cabeça e pescoço. Radiologia Brasileira. 2011; 44(5): $297-300$ 